\title{
Relationship between neutrophils to HDL-C ratio and severity of coronary stenosis
}

\author{
Tuli Kou ${ }^{1 \dagger}$, Haorou Luo ${ }^{2 \dagger}$ and Lixue Yin ${ }^{2,3 *}$ (1)
}

\begin{abstract}
Background: Lipid and inflammatory molecules play a key role in the development of inflammation. Neutrophil counts are used as markers of inflammation duration, and HDL-C is used as an anti-atherosclerosis component. However, few studies have been found to integrate these two indicators to explore coronary stenosis. We suggested that neutrophil count as a marker of inflammation persistence and HDL-C as an anti-atherosclerotic component should be integrated into a single biomarker NHR to explore its correlation with CAD degree and predict the severity of coronary stenosis among CAD patients.
\end{abstract}

Methods: We examined 404 eligible patients who underwent coronary angiography. Based on the results of coronary angiography, patients in $C A D^{+}$group $(n=155)$ were defined as those having angiographic coronary stenosis of at least 50\% lumen reduction in at least one major coronary artery (including left anterior descending artery, left circumflex artery, left main coronary artery, right coronary artery). Patients with luminal stenosis but no more than $50 \%$ were defined as $C A D^{-}$group $(n=49)$, and patients without luminal stenosis $(n=200)$ were regarded as control group. The relationship between various serum markers and the severity of coronary stenosis was examined by Spearman correlation analysis. Logistic regression analysis was performed to identify the influencing factors of the severity of coronary artery disease.

Results: The modified Gensini score was positively correlated with neutrophil HDL-C ratio and negatively correlated with albumin and $\mathrm{HDL}-\mathrm{C}$. Multiple regression analysis showed that neutrophil $\mathrm{HDL}-\mathrm{C}$ ratio were significantly associated with CAD. Neutrophil HDL-C ratio is an independent predictor of CAD. The ROC analysis provided a cut-off value of 1.51 for neutrophil HDL-C ratio to predict CAD with $94.8 \%$ sensitivity and 0.024 Yoden index, and area under the ROC curve of 0.617 (95\% Cl 0.560-0.675, $P<0.001)$.

Conclusion: Neutrophil HDL-C ratio is not only closely related to coronary artery stenosis, but also an independent predictor of severe coronary stenosis.

Keywords: Neutrophil, High-density lipoprotein cholesterol, Coronary stenosis, Coronary artery disease

*Correspondence: yinlixue_cardiac@163.com

${ }^{\dagger}$ Tuli Kou and Haorou Luo contributed equally to this work. They should be considered co-first authors

${ }^{2}$ School of Medicine, University of Electronic Science and Technology

of China, Chengdu, Sichuan, China

Full list of author information is available at the end of the article

\section{Background}

Coronary artery disease (CAD) is a catastrophic disease with high morbidity and mortality rates [1], causing adverse cardiac events in severe cases. CAD has the highest mortality rate in developed countries [2] and is now prevalent in developing countries as well; thus, it is becoming the primary cause of mortality worldwide [3]. Atherosclerosis plays a dominant role in the pathophysiological process of the disease, and studies have shown that $\mathrm{CAD}$ and atherosclerosis are closely associated with 
inflammation [1]. Meanwhile, lipid and inflammatory molecules play a key role in the development of inflammation. Current studies have shown that neutrophils contribute critically in the atherosclerotic process [4] and are markers of persistent inflammation as well as predictors of cardiovascular risk [5]. Moreover, high-density lipoprotein cholesterol (HDL-C) has been found to be closely related to coronary artery stenosis; it has the function of not only reverse cholesterol transport but also oxidation resistance and vascular endothelial function protection $[6,7]$,with its concentration being negatively correlated with the coronary heart disease risk [8]. Neutrophils and HDL-C are both important in the atherosclerotic process. However, few studies have been found to integrate these two indicators. Therefore, this study suggested that neutrophil count as a marker of inflammation persistence and HDL-C as an anti-atherosclerotic component should be integrated into a single biomarker NHR to explore its correlation with CAD degree and predict the severity of coronary stenosis among CAD patients [9].

\section{Methods}

\section{Study population}

This was a retrospective study consisting of 404 consecutive patients (174 males, 43.1\%), who had undergone coronary angiography at the Sichuan Provincial People's Hospital from June 2018 to January 2020 due to CADrelated symptoms such as typical chest pain. All patients with coronary stent implantation, acute coronary Syndrome (ACS), coronary artery bypass surgery, liver and kidney disease, inflammatory disease, heart failure, blood diseases, rheumatic diseases, malignant tumors, alcohol use and antioxidant drugs (including statins, beta blockers, and aspirin) were excluded. Based on the results of coronary angiography, patients in $\mathrm{CAD}^{+}$group $(\mathrm{n}=155)$ were defined as those having angiographic coronary stenosis of at least $50 \%$ lumen reduction in at least one major coronary artery (including left anterior descending artery, left circumflex artery, left main coronary artery, right coronary artery).Patients with luminal stenosis but no more than $50 \%$ were defined as $\mathrm{CAD}^{-}$group $(\mathrm{n}=49)$, and patients without luminal stenosis $(\mathrm{n}=200)$ were regarded as control group. Specifically, we did not include patients with diffuse coronary artery involvement but no stenosis. At the same time, when measuring the degree of obstuction on lumenogram, two experienced interventional cardiologists made a comprehensive judgment. The current study was in line with the principles of the Declaration of Helsinki.

\section{Clinical data and definitions}

Height and weight were measured to calculate body mass index (BMI), which was counted as weight divided by height squared, weight in kilograms and height in meters. Collection of serum biomarkers such as uric acid, creatinine, triglycerides, bilirubin, total cholesterol, high-density lipoprotein cholesterol (HDL-C), albumin, low-density lipoprotein cholesterol (LDL-C), neutrophils, and eosinophils. Serum total antioxidant status of was calculated as: $(0.63 \times$ albumin concentration $)+(1.53 \times$ bilirubin concentration $)+(1.02 \times$ uric acid concentration), where the concentrations of uric acid, bilirubin and albumin were expressed in $\mathrm{mmol} / \mathrm{L}$. Neutrophil and HDL-C ratios were calculated. Hypertension was defined as blood pressure 140/90 $\mathrm{mmHg}$ or higher, or they were being given antihypertensive drugs. Those who had been diagnosed with hypertension in the past were all classified as having a history of hypertension. Patients were considered to have diabetes if they had a previous history of diabetes, or plasma glucose-lowering drugs use, including oral tablets and insulin. Current smokers were defined as those who smoked at least one cigarette a day for more than one year in a row; Longterm smokers who have quit for less than 6 months were still considered to have a smoking history.

\section{Gensini score system}

The modified Gensini score (GS) system was used to measure CAD severity. These scores were expressed as the sum of the positional score of each lesion multiplied by the obstruction severity score, which emphasizes the severity of the disease. The calculation of the modified Gensini score system is shown in Table 1.

\section{Statistical analysis}

All statistical analyses were performed using SPSS 25.0 for Mac. Kolmogorov-Smirnov test was used to verify that the continuous variable was normally distributed. The continuous variables of normal distribution were indicated as the mean \pm standard deviation. Nonnormally distributed variables were represented as medians. Categorical variables were expressed in terms of frequency percentages and absolute numbers. Homogeneity of variance was evaluated by Levene's test. If the data for continuous variables satisfied normal distribution and homogeneity of variance, a one-way analysis of variance was used, and the comparison among groups were performed with LSD method; Otherwise, the KruskalWallis test was used to compare the differences of the non-conforming measurement data. Categorical variables were compared using the chi-squared. Correlation of Gensini score with serum biomarkers was analyzed by Pearson or Spearman's correlation coefficient, as appropriate. Before further statistical analysis, Spearman's correlation coefficients were first calculated to eliminate multicollinearity between or among variables. To determine the risk factors associated with $\mathrm{CAD}$, multiple 
Table 1 Gensini score system

\begin{tabular}{lll}
\hline The degree of stenosis (\%) & Score & Lesion location \\
\hline $1-25$ & 1 & The second diagonal and other segments \\
$26-50$ & 2 & Right coronary artery \\
$51-75$ & 4 & The mid-distal region of the left circumflex artery \\
$76-90$ & 8 & The distal left anterior descending artery, first diagonal branch \\
$91-99$ & 16 & The mid-region of the left anterior descending artery \\
100 & 32 & The proximal left anterior descending artery or proximal left circumflex artery \\
& & The left main coronary artery
\end{tabular}

unconditional logistic regression models were performed as the primary analysis for the independent effects of each variable. Univariate logistic regression models were first performed to explore the crude association between the severity of coronary stenosis and each factor, including the ratio of neutrophils to high-density lipoprotein (NHR), sex, age, smoking, hypertension, albumin, creatinine, uric acid, HDL cholesterol, LDL cholesterol, and neutrophils individually. Factors that were significant in the univariate model at the $\mathrm{p}<0.10$ level were contained in the multivariate logistic regression model, so as to identify factors independently associated with the presence of coronary artery stenosis. Using the backward step-by-step approach, All statistically significant factors, i.e. $\mathrm{P}<0.05$ level, were contained in the final prediction model. A receiver operating characteristic (ROC) curve was presented. Regression coefficients and 95\% confidence intervals (CIs) for risk factors significantly associated with CAD were calculated. All statistical tests were double-sided, and $\mathrm{P}$ value $<0.05$ was considered statistically significant.

\section{Results}

\section{Baseline characteristics}

A total of 404 patients were included in our analysis. Demographic and clinical patient characteristics are presented in Table 2. The smoking incidence, hypertension incidence, creatinine, and neutrophil levels

Table 2 Clinical and laboratory characteristics of patients

\begin{tabular}{|c|c|c|c|c|}
\hline Parameters & $\mathrm{CAD}^{+}$group $(n=155)$ & $C A D^{-}$group $(n=49)$ & Control group $(n=200)$ & $\mathbf{P}$ \\
\hline Age (year) & $64.57 \pm 10.57$ & $64.22 \pm 10.10$ & $59.24 \pm 10.69$ & $<0.001$ \\
\hline Male [n (\%)] & $85(54.8)$ & $22(44.9)$ & $67(33.5)$ & $<0.001$ \\
\hline Body mass index (kg/m) & $24.43 \pm 3.21$ & $24.19 \pm 3.21$ & $23.74 \pm 4.13$ & 0.212 \\
\hline Smoking $[\mathrm{n}(\%)]$ & $46(29.7)$ & $8(16.3)$ & $30(15.0)$ & 0.002 \\
\hline Hypertension [n (\%)] & $78(50.3)$ & $22(44.9)$ & $70(35.0)$ & 0.014 \\
\hline Diabetes mellitus [n (\%)] & $35(22.6)$ & $9(18.4)$ & $26(13.0)$ & 0.06 \\
\hline Creatinine $(\mu \mathrm{m} / \mathrm{L})$ & $68.99 \pm 26.60$ & $65.90 \pm 12.23$ & $61.53 \pm 14.08$ & 0.009 \\
\hline Albumin (g/L) & $41.76 \pm 5.06$ & $43.91 \pm 4.04$ & $42.77 \pm 3.61$ & 0.001 \\
\hline Total bilirubin $(\mu \mathrm{m} / \mathrm{L})$ & $15.11 \pm 6.81$ & $16.24 \pm 7.21$ & $14.91 \pm 6.17$ & 0.403 \\
\hline Direct bilirubin ( $\mu \mathrm{m} / \mathrm{L})$ & $5.01 \pm 3.81$ & $5.00 \pm 1.37$ & $5.00 \pm 1.41$ & 0.102 \\
\hline Indirect bilirubin $(\mu \mathrm{m} / \mathrm{L})$ & $10.26 \pm 5.42$ & $11.23 \pm 6.10$ & $9.97 \pm 5.26$ & 0.269 \\
\hline Total cholesterol (mmol/L) & $4.92 \pm 1.13$ & $4.70 \pm 0.95$ & $4.74 \pm 1.01$ & 0.236 \\
\hline Triglyceride (mmol/L) & $2.23 \pm 1.74$ & $1.96 \pm 1.19$ & $2.14 \pm 1.75$ & 0.429 \\
\hline Uric acid $(\mu m / L)$ & $328.74 \pm 83.86$ & $316.10 \pm 93.58$ & $318.50 \pm 74.07$ & 0.455 \\
\hline LDL-C (mmol/L) & $2.82 \pm 0.92$ & $2.67 \pm 0.82$ & $2.62 \pm 0.80$ & 0.119 \\
\hline $\mathrm{HDL}-\mathrm{C}(\mathrm{mmol} / \mathrm{L})$ & $1.28 \pm 0.43$ & $1.30 \pm 0.29$ & $1.35 \pm 0.33$ & 0.023 \\
\hline Neutrophils $\left(\times 10^{9} / \mathrm{L}\right)$ & $4.67 \pm 2.25$ & $3.92 \pm 1.63$ & $4.08 \pm 0.50$ & 0.032 \\
\hline Eosinophil $\left(\times 10^{9} / \mathrm{L}\right)$ & $0.94 \pm 7.26$ & $0.10 \pm 0.09$ & $0.11 \pm 0.12$ & 0.273 \\
\hline cTAS (mmol/L) & $0.75 \pm 0.10$ & $0.76 \pm 0.11$ & $0.75 \pm 0.09$ & 0.592 \\
\hline Gensini score & $18(24.8-34.9)$ & $3(3.5-6.1)$ & 0 & $<0.001$ \\
\hline NHR & $3.99 \pm 2.16$ & $3.20 \pm 1.75$ & $3.24 \pm 1.78$ & $<0.001$ \\
\hline
\end{tabular}


in $\mathrm{CAD}^{+}$group were apparently higher than that in $\mathrm{CAD}^{-}$group and control group $(\mathrm{P}<0.05$, respectively). Compared to the $\mathrm{CAD}^{-}$group, the $\mathrm{CAD}^{+}$group showed a significantly higher Gensini score $(\mathrm{P}<0.05)$. Nevertheless, the levels of HDL-C and albumin in the

Table 3 Correlation coefficient between risk factors and Gensini score

\begin{tabular}{lcc}
\hline Parameters & $\mathbf{r}$ & $\mathbf{P}$ \\
\hline NHR and Gensini score & 0.225 & $<0.001$ \\
CTAS and Gensini score & 0.013 & 0.788 \\
Neutrophils and Gensini score & 0.128 & 0.01 \\
Eosinophil and Gensini score & -0.044 & 0.383 \\
Uric acid and Gensini score & 0.073 & 0.145 \\
Creatinine and Gensini score & 0.189 & $<0.001$ \\
Albumin and Gensini score & -0.13 & 0.009 \\
Total bilirubin and Gensini score & 0.03 & 0.551 \\
Direct bilirubin and Gensini score & -0.064 & 0.2 \\
Indirect bilirubin and Gensini score 0.06 & 0.058 & 0.248 \\
Total cholesterol and Gensini score & 0.071 & 0.153 \\
Triglyceride and Gensini score & 0.058 & 0.245 \\
LDL-C and Gensini score & 0.113 & 0.023 \\
HDL-C and Gensini score & -0.189 & $<0.001$ \\
Sex and Gensini score & 0.143 & 0.004 \\
Age and Gensini score & 0.216 & $<0.001$ \\
Cigarette smoking and Gensini score & 0.117 & 0.019 \\
Hypertension and Gensini score & 0.104 & 0.036 \\
Diabetes mellitus and Gensini score & 0.049 & 0.323 \\
\hline
\end{tabular}

$\mathrm{CAD}^{+}$group were lower than those in the $\mathrm{CAD}^{-}$group $(\mathrm{P}<0.05)$. There were statistically significant differences in age and gender among the three groups $(\mathrm{P}<0.05)$, and the average age of patients in the $\mathrm{CAD}^{+}$group was higher than that in the $\mathrm{CAD}^{-}$group $(\mathrm{P}<0.001)$. The proportion of males in the $\mathrm{CAD}^{+}$group was apparently higher than that in the $\mathrm{CAD}^{-}$group and control group $(\mathrm{P}<0.05$, respectively). There were no significant differences in BMI, diabetes mellitus,cTAS,eGFR ,bilirubin,total cholesterol, triglycerides,uric acid, and LDL-C, eosinophils among the three groups $(P>0.05$, respectively).

Relationship between risk factors and the modified Gensini score

To clarify the relationship between various risk factors and the modified Gensini score in patients with coronary atherosclerotic heart disease, Spearman rank correlation analysis was conducted. As shown in Table 3. The results showed that the Gensini score was positively correlated with NHR, neutrophils, creatinine, LDL-C, sex, age, cigarette smoking, hypertension, there was a negative correlation between Gensini score and HDL-C, as well as albumin. while Gensini score was not correlated with cTAS, eosinophil, uric acid, bilirubin, total cholesterol, triglyceride, and diabetes mellitus. The scatter diagram is shown in Fig. 1.

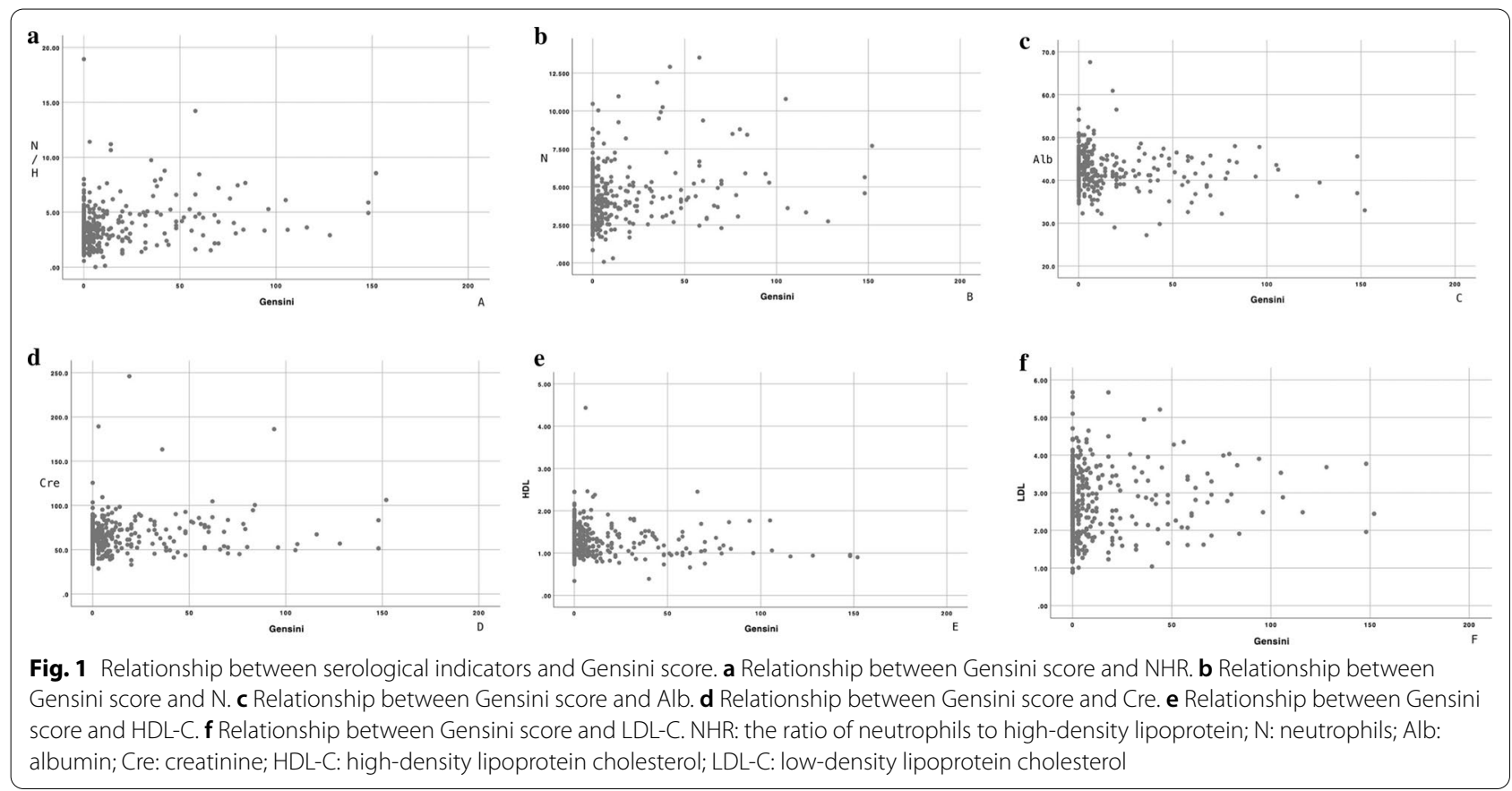


Univariate and multivariate logistic regression analyses To further explore the possible risk factors for coronary artery stenosis, univariate and multivariate logistic regression analyses were applied (Table 4). Univariate Logistic regression analyses showed that risk factors associated with CAD included NHR, smoking, hypertension, sex, age, creatinine, albumin, LDL-C, and central granulocyte $(\mathrm{p}<0.05)$. Additionally, after adjusting for all covariates, multiple regression analyses showed that NHR, age, sex, albumin, and LDL-C were significantly correlated with CAD. Consequently, NHR was an independent predictor of CAD.The ROC analysis displayed that the critical value of NHR was $1.51,94.8 \%$ of sensitivity and 0.024 of Yoden index could predict CAD, and the area under the ROC curve was 0.617 (95\% CI 0.5600.675, $\mathrm{P}<0.001$, Fig. 2).

\section{Discussion}

The results of this study showed that elevated NHR levels were closely correlated with the degree of coronary artery stenosis and that NHR was an independent predictor of CAD. Our study appears to be novel in that the use of NHR, which is a simple and readily available inflammatory marker, may help predict CAD and assess coronary artery stenosis.

Despite ongoing efforts to manage disease, such as strengthening medical care and advancements in invasive procedures, CAD remains the leading cause of death in patients with cardiovascular disease [10]. In patients presenting with chest pain, assessing the severity of coronary stenosis is critical in determining optimal treatment strategies and associated cardiovascular risk stratification [11]. The pathophysiological processes of CAD are complicated, which include altered lipid metabolism, inflammatory reaction, oxidative injury and other processes, in which various inflammatory factors are playing a central role $[12,13]$. In this study, we hypothesised that NHR

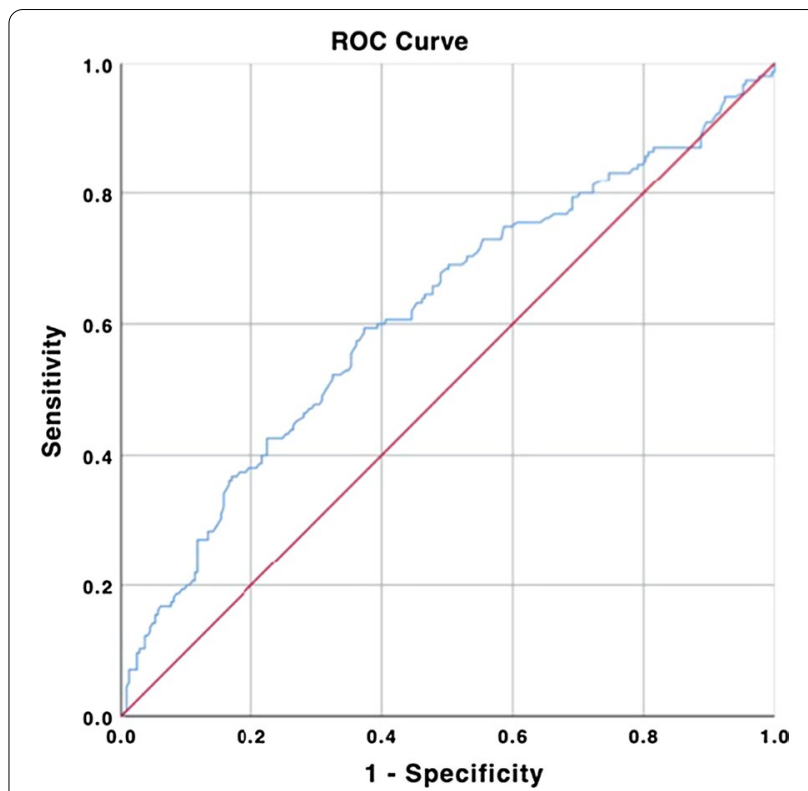

Fig. 2 Receiver-operating characteristic curve analysis for NHR for prediction of CAD

was a new indicator composed of inflammatory cells and lipid cholesterol that might reflect inflammation status and lipid metabolism more comprehensively than a single biological indicator. In the univariate and multivariate analyses, we investigated a number of factors associated with $\mathrm{CAD}$, and the results showed that the Gensini score was positively correlated with NHR. After adjusting for confounding factors, NHR was found to be an independent predictor of CAD. Moreover, this study found that the NHR cutoff of 1.5 had a sensitivity of $94.8 \%$ in predicting artery disease.

Based on our analysis, we attempted to explain the association between higher NHR and severe coronary stenosis. Neutrophils are important components

Table 4 Logistic regression analysis of factors associated with the presence of CAD

\begin{tabular}{|c|c|c|c|c|}
\hline \multirow[t]{2}{*}{ Variables } & \multicolumn{2}{|l|}{ Univariate } & \multicolumn{2}{|l|}{ Multivariate } \\
\hline & OR $(95 \% \mathrm{Cl})$ & $\mathbf{P}$ & OR $(95 \% \mathrm{Cl})$ & $\mathbf{P}$ \\
\hline Creatinine & $1.020(1.008-1.033)$ & 0.001 & $1.004(0.992-1.016)$ & 0.591 \\
\hline Albumin & $0.921(0.874-0.970)$ & 0.002 & $0.944(0.893-0.998)$ & 0.043 \\
\hline $\mathrm{NHR}$ & $1.232(1.098-1.381)$ & $<0.001$ & $1.163(1.034-1.308)$ & 0.012 \\
\hline Sex & $2.183(1.450-3.286)$ & $<0.001$ & $2.664(1.668-4.255)$ & $<0.001$ \\
\hline Age & 1.039 (1.019-1.060) & $<0.001$ & $1.048(1.025-1.071)$ & $<0.001$ \\
\hline LDL & $1.281(1.013-1.620)$ & 0.039 & $1.460(1.130-1.886)$ & 0.004 \\
\hline Neutrophils & $1.204(1.075-1.348)$ & 0.001 & $1.010(0.821-1.244)$ & 0.82 \\
\hline Cigarette smoking & $2.343(1.439-3.817)$ & 0.001 & $1.671(0.900-3.104)$ & 0.096 \\
\hline Hypertension & $1.729(1.151-2.596)$ & 0.008 & $1.407(0.894-2.214)$ & 0.107 \\
\hline
\end{tabular}


involved in immune inflammation [14, 15]. Previous studies have shown abundance of neutrophils in coronary artery lesions [16]. Recent evidences have suggested that elevated level of myeloperoxidase from neutrophils could contribute to coronary atherosclerosis [17]. Furthermore, neutrophils were associated with the complexity of coronary stenosis and were independent predictors of multiple complex stenoses [5]. Our results showed that the neutrophil level in the $\mathrm{CAD}^{+}$group was higher than that in the coronary atherosclerosis group and control group, and the Gensini score was positively correlated with neutrophils, which was also consistent with the results of previous studies [18].

Dyslipidemia may account for $50 \%$ of the attributable risk in CAD population, so recent studies appear to pay more attention to the association between lipid-related biomarkers and coronary disease [19]. HDL-C, as a typical lipid-related biomarker, plays an important protective role in atherosclerosis and inflammation. Its mechanism of action was to transport excess cholesterol from peripheral tissues back to the liver for excretion. Meanwhile, it can inhibit the expression of endothelial cell adhesion molecules and prevent monocytes from accumulating into the arterial wall $[20,21]$. More importantly, HDL-C inhibits neutrophil activation, diffusion and migration, which may be associated with lipid raft abundance [22].

Above all, because of the interaction of decreased HDL-C level and increased neutrophil count in CAD, an elevated NHR might be related to abnormal lipid metabolism and inflammatory activity. Furthermore, as regards the complex interaction between neutrophils and HDL-C, a comprehensive indicator of NHR may be more effective and reliable than a single indicator. In previous studies, NHR was calculated as a simple ratio of neutrophils and HDL-C, and related reports have assessed them as predictive markers of metabolic syndrome [23]. Another study showed a correlation between NHR and the extent of coronary artery disease [24]. However, that study focused on acute myocardial infarction, and our study was designed to directly discuss problems related to the severity of coronary artery lesions in CAD. In addition, we performed regression analysis of predictors of multiple indicators. Our large sample size was our advantage. Foremost, as a new predictor of CAD, NHR could be calculated from the complete blood count on admission, which is a fast and convenient method.

The Gensini score is an effective marker for assessing CAD severity. Coronary artery branches with varying severities have different weight coefficients, which can reflect disease severity more objectively $[25,26]$. Previous studies have shown that Gensini scores were used as basis of grouping patients with severe CAD, and clear differences were found between the high score groups and other groups [27-29]. According to our results, NHR is positively correlated with Gensini scores. The combination of NHR and Gensini scores could help clinicians more effectively identify high-risk patients with coronary artery stenosis. In addition, relevant reports have suggested that cTAS is closely related to the severity of coronary stenosis $[28,29]$. The expanded sample size test found that it was inconsistent with our results, possibly because the serum cTAS measured in the circulating blood may not be a true indicator of antioxidant concentrations in atherosclerotic plaques, and the equation for calculating cTAS in our study was neither very sensitive nor specific. Maryam et al. did not find that the cTAS level was an independent predictor of CAD, which was also consistent with our results [29].

In conclusion, we believe that NHR may be a widely used biomarker to predict $\mathrm{CAD}$ and assess the degree of coronary stenosis. However, our study had some limitations. First, this was a single-centre retrospective study, so there were limitations in persuasiveness. Furthermore, we did not explore the association of CRP, neopterin and other inflammatory markers with CAD. Finally, we did not explore the correlation between NHR and MACE. In future work, we will expand the sample size for further investigation.

\section{Conclusion}

We found that NHR is not only closely related to coronary artery stenosis, but also an independent predictor of severe coronary artery stenosis and can be used as an independent indicator of the severity of coronary artery stenosis. Unlike many other biometrics, NHR is a relatively inexpensive and readily available marker that can provide effective value in predicting the severity of coronary stenosis (Additional file 1).

\section{Supplementary information}

Supplementary information accompanies this paper at https://doi. org/10.1186/s12872-020-01771-z.

Additional file 1: Figure 1. Relationship between serological indicators and Gensini score.

\section{Abbreviations}

NHR: Neutrophils to high density lipoprotein cholesterol ratio; CAD: Coronary artery disease; HDL-C: High density lipoprotein cholesterol; LDL-C: Low density lipoprotein cholesterol; ACS: Acute coronary syndrome; BMI: Body mass index; GS: Gensini score system; ROC: A receiver operating characteristic; $\mathrm{Cl}$ : Confidence intervals; AMI: Acute myocardial infarction; LSD: Least significant difference; cTAS: The levels of calculated serum total antioxidant status; eGFR: Estimated glomerular filtration rate.

\section{Acknowledgements}

The authors express their gratitude to Sichuan provincial people's hospital for their cooperation and assistance. 


\section{Authors' contributions}

$\mathrm{HL}$ and TK meet the International Committee of Medical Journal Editors (ICMJE) criteria for authorship of this article, take responsibility for the integrity of the work as a whole, and have given their approval for this version to be published. $\mathrm{HL}$ and TK conceptualized and designed the study. $\mathrm{HL}$ and TK verified, analyzed, and interpreted the data. HL and TK wrote the manuscript and substantially contributed to critical revision of the intellectual content. $\mathrm{HL}$ and TK contributed equally to this work. They should be considered co-first authors. LY, the corresponding author of this paper, interpreted the data and substantially contributed to critical revision of the intellectual content. All authors read and approved the final manuscript.

\section{Funding}

Not applicable.

\section{Availability of data and materials}

All data are freely available for scientific purpose. The data can be found from all listed authors.

\section{Ethics approval and consent to participate}

This retrospective database analysis did not involve the collection, use, or transmittal of individual identifiable data. As such, Institutional Review Board (IRB) approval to conduct this study was not required and considered exempt according to 45CFR46.101(b)(4): Existing Data \& Specimens-No Identifiers. Both the data set itself and the security of the offices where the data are housed meet the requirements of the Health Insurance Portability and Accountability Act (HIPAA) of 1996. Administrative permissions were required to access the raw data from Sichuan Provincial People's Hospital.With the permission of Sichuan Provincial People's Hospital and the department of Cardiovascular Medicine, we were allowed to access the raw data of Sichuan Provincial People's Hospital.

\section{Consent for publication}

Not applicable.

\section{Competing interests}

The authors declare that they have no competing interests.

\section{Author details}

1 Southwest Medical University, Luzhou, China. ${ }^{2}$ School of Medicine, University of Electronic Science and Technology of China, Chengdu, Sichuan, China. ${ }^{3}$ Sichuan Academy of Medical Sciences, Sichuan Provincial People's Hospital, Chengdu, China

Received: 16 July 2020 Accepted: 8 November 2020

Published online: 06 March 2021

\section{References}

1. Akyel A, Yayla Ç, Erat M, et al. Neutrophil-to-lymphocyte ratio predicts hemodynamic significance of coronary artery stenosis. Anatol J Cardiol. 2015;15(12):1002-7.

2. Shahsavari G, Raoufi A, Toolabi A, Hosseninejadmir N, Ahmadvand H, Safariebrahimsarabie $\mathrm{M}$. The effect of atorvastatin treatment duration on oxidative stress markers and lipid profile in patients with coronary artery diseases: a case series study. ARYA Atheroscler. 2017;13(6):282-7.

3. Guo T, Huang L, Liu C, et al. The clinical value of inflammatory biomarkers in coronary artery disease: PTX3 as a new inflammatory marker. Exp Gerontol. 2017;97:64-7.

4. Güven R, Akyol KC, Bayar N, Güngör F, Akça AH, Çelik A. Neutrophil count as a predictor of critical coronary artery stenosis in young patients. Iran J Public Health. 2018;47(5):765-7.

5. Avanzas P, Arroyo-Espliguero R, Cosín-Sales J, Quiles J, Zouridakis E, Kaski JC. Multiple complex stenoses, high neutrophil count and C-reactive protein levels in patients with chronic stable angina. Atherosclerosis. 2004;175(1):151-7.

6. Li Y, Jin P, Hou F, Zhou Y. Association between TG-to-HDL-C ratio and instent stenosis under optical coherence tomography guidance. J Med Syst. 2018;43(1):4.
7. Sun T, Hu J, Yin Z, et al. Low serum paraoxonase1 activity levels predict coronary artery disease severity. Oncotarget. 2017;8(12):19443-54.

8. Chen Y, Dong J, Chen $X$, et al. Human serum preß1-high density lipoprotein levels are independently and negatively associated with coronary artery diseases. Nutr Metab (Lond). 2016;13:36.

9. Yılmaz S, Canpolat U, Başer K, Ünal S, Kuyumcu MS, Aydoğdu S. Neutrophil-to-lymphocyte ratio predicts functionally significant coronary artery stenosis in patients with stable coronary artery disease. Turk Kardiyol Dern Ars. 2018;46(2):129-35.

10. Chan MY, Du X, Eccleston D, et al. Acute coronary syndrome in the Asia-Pacific region. Int J Cardiol. 2016;202:861-9.

11. Mor-Avi V, Patel MB, Maffessanti F, et al. Fusion of Three-dimensional echocardiographic regional myocardial strain with cardiac computed tomography for noninvasive evaluation of the hemodynamic impact of coronary stenosis in patients with chest pain. J Am Soc Echocardiogr. 2018;31(6):664-73.

12. Hong MK, Mintz GS, Lee CW, et al. Comparison of coronary plaque rupture between stable angina and acute myocardial infarction: a three-vessel intravascular ultrasound study in 235 patients. Circulation. 2004:110(8):928-33.

13. Incalcaterra E, Accardi G, Balistreri CR, et al. Pro-inflammatory genetic markers of atherosclerosis. Curr Atheroscler Rep. 2013;15(6):329.

14. Montecucco F, Liberale L, Bonaventura A, Vecchiè A, Dallegri F, Carbone F. The role of inflammation in cardiovascular outcome. Curr Atheroscler Rep. 2017;19(3):11.

15. Ding $S$, Lin N, Sheng $X$, et al. Melatonin stabilizes rupture-prone vulnerable plaques via regulating macrophage polarization in a nuclear circadian receptor RORa-dependent manner. J Pineal Res. 2019;67(2):e12581.

16. Soehnlein O, Weber C. Myeloid cells in atherosclerosis: initiators and decision shapers. Semin Immunopathol. 2009;31(1):35-47.

17. Wang XS, Kim HB, Szuchman-Sapir A, McMahon A, Dennis JM, Witting PK. Neutrophils recruited to the myocardium after acute experimental myocardial infarct generate hypochlorous acid that oxidizes cardiac myoglobin. Arch Biochem Biophys. 2016;612:103-14.

18. Chen J, Chen MH, Li S, et al. Usefulness of the neutrophil-to-lymphocyte ratio in predicting the severity of coronary artery disease: a Gensini score assessment. J Atheroscler Thromb. 2014;21(12):1271-82.

19. Song $Y$, Yang $Y$, Zhang J, et al. The apoB100/apoAl ratio is independently associated with the severity of coronary heart disease: a cross sectional study in patients undergoing coronary angiography. Lipids Health Dis. 2015:14:150.

20. Ghattas A, Griffiths HR, Devitt A, Lip GY, Shantsila E. Monocytes in coronary artery disease and atherosclerosis: where are we now? J Am Coll Cardiol. 2013;62(17):1541-51.

21. Rohatgi A. High-density lipoprotein function measurement in human studies: focus on cholesterol efflux capacity. Prog Cardiovasc Dis. 2015;58(1):32-40.

22. Murphy AJ, Woollard KJ, Suhartoyo A, et al. Neutrophil activation is attenuated by high-density lipoprotein and apolipoprotein A-I in in vitro and in vivo models of inflammation. Arterioscler Thromb Vasc Biol. 2011;31(6):1333-41.

23. Chen $\mathrm{T}$, Chen $\mathrm{H}_{\text {, Xiao }} \mathrm{H}_{\text {, et }}$ al. Comparison of the value of neutrophil to high-density lipoprotein cholesterol ratio and lymphocyte to high-density lipoprotein cholesterol ratio for predicting metabolic syndrome among a population in the Southern Coast of China. Diabetes Metab Syndr Obes. 2020;13:597-605.

24. Huang JB, Chen YS, Ji HY, et al. Neutrophil to high-density lipoprotein ratio has a superior prognostic value in elderly patients with acute myocardial infarction: a comparison study. Lipids Health Dis. 2020;19(1):59.

25. İscanlı MD, Metin Aksu N, Evranos B, Aytemir K, Özmen MM. Comparison of TIMI and Gensini score in patients admitted to the emergency department with chest pain, who underwent coronary angiography. Med Sci Monit. 2014;20:343-9.

26. Rampidis GP, Benetos G, Benz DC, Giannopoulos AA, Buechel RR. A guide for Gensini Score calculation. Atherosclerosis. 2019;287:181-3.

27. Nose D, Shiga Y, Ueda Y, et al. Association between plasma levels of PCSK9 and the presence of coronary artery disease in Japanese. Heart Vessels. 2019:34(1):19-28. 
28. Turan T, Menteşe Ü, Ağaç MT, et al. The relation between intensity and complexity of coronary artery lesion and oxidative stress in patients with acute coronary syndrome. Anatol J Cardiol. 2015;15(10):795-800.

29. Sotoudeh Anvari M, Mortazavian Babaki M, Boroumand MA, Eslami B, Jalali A, Goodarzynejad H. Relationship between calculated total antioxidant status and atherosclerotic coronary artery disease. Anatol J Cardiol. 2016;16(9):689-95.

\section{Publisher's Note}

Springer Nature remains neutral with regard to jurisdictional claims in published maps and institutional affiliations.
Ready to submit your research? Choose BMC and benefit from:

- fast, convenient online submission

- thorough peer review by experienced researchers in your field

- rapid publication on acceptance

- support for research data, including large and complex data types

- gold Open Access which fosters wider collaboration and increased citations

- maximum visibility for your research: over $100 \mathrm{M}$ website views per year

At BMC, research is always in progress.

Learn more biomedcentral.com/submissions 